\title{
CONHECIMENTO DE ESTUDANTES DE MEDICINA SOBRE DOAÇÃO E TRANSPLANTES DE ÓRGÃOS
}

\author{
Knowledge of medical students on organ donation and transplantation
}

\author{
Carla Reale Batista1', Liliane Elze Falcão Lins Kusterer²
}

\begin{abstract}
RESUMO
Objetivo: Avaliar o conhecimento de estudantes de Medicina sobre doação e transplantes de órgãos e o conceito de morte encefálica, além de identificar os possíveis fatores contrários à doação de órgãos e tecidos. Métodos: Trata-se de um estudo de corte transversal realizado com 266 estudantes de Medicina do primeiro ao quarto ano de graduação. A coleta de dados foi realizada entre agosto e outubro de 2010 através da aplicação de um questionário contendo dados demográficos e 13 perguntas de múltipla escolha sobre o tema. Resultados: Dentre os entrevistados, $61,3 \%$ tiveram atividades didáticas sobre transplantes de órgãos durante o curso. Sessenta por cento dos estudantes avaliaram o seu conhecimento, sobre o tema como regular. Oitenta e oito por cento dos estudantes acertaram o conceito de morte encefálica e $72 \%$ tinham noções de como realizar o diagnóstico. Em relação à quais transplantes poderiam ser realizados intervivos, $78,9 \%$ responderam corretamente. Já com relação aos transplantes realizados sem batimentos cardíacos, 45,4\% acertaram a questão e 53,4\% não souberam responder. Quando perguntados sobre a intenção de doar órgãos e tecidos, 78,6\% eram doadores, sendo que a análise por semestre mostrou aumento progressivo na intenção de doar. Com relação aos cuidados que devem ser tomados para a manutenção do potencial doador de órgãos, 75,5\% dos estudantes desconheciam as medidas de suporte necessárias. No que se refere à comunicação familiar da morte de um parente, 50,4\% sabiam como era feita essa abordagem. Conclusão: Foi possível concluir que a maioria dos estudantes de Medicina da amostra estudada apresentou conhecimento sobre morte encefálica, e que durante o curso médico os estudantes adquirem maior conhecimento sobre transplante e doação de órgãos e tecidos. Esse conhecimento adquirido aumenta a intenção de doar, sugerindo que a educação seja imprescindível para melhorar as taxas de doação de órgãos. Dessa forma, é necessária inserção de matérias ou cursos específicos de doação de órgãos e tecidos para transplantes no currículo das faculdades de Medicina.
\end{abstract}

Descritores: Transplantes, Morte Encefálica, Estudantes de Medicina, Doação de Órgão, Doação de Tecido.

\section{Instituições:}

${ }^{1}$ Acadêmica de Medicina da EBMSP, Salvador, BA, Brasil.

${ }^{2}$ Departamento de Biomorfologia da Escola Bahiana de Medicina e Saúde Pública (EBMSP), Salvador, BA, Brasil.

\section{Correspondência:}

Dra. Liliane Lins,

Escola Bahiana de Medicina e Saúde Pública.

Rua Frei Henrique, $n^{\circ}$ 8, CEP 40050-420, Salvador-BA, Brasil.

Fax.: (71) 3241-6164

E-mail: liliane@linsreabilitacao.com.br.

\section{INTRODUÇÃO}

Atualmente, há a nível mundial uma discrepância entre a quantidade de potenciais doadores de órgãos e tecidos e o número de receptores. ${ }^{1}$ Em todo o mundo, mais de $20 \%$ dos pacientes na lista de espera para transplantes morrem anualmente devido à escassez de doadores de órgãos. ${ }^{2}$ No Brasil, cerca de 64.000 indivíduos se encontram em lista de espera, sendo que a demora no atendimento desses pacientes pode ter impacto negativo sobre a evolução da doença e sobrevida dos mesmos. ${ }^{3,4}$

A capacitação de futuros profissionais de saúde na área de transplante de órgãos é fundamental para o aumento do número de doações, além de otimizar as etapas necessárias para a realização de transplante, aumentando o número de notificações de potenciais doadores, o índice de captação e a qualidade dos enxertos. As escolas médicas, em sua maioria, não apresentam formação específica para doação de órgãos e transplantes, desconsiderando o papel fundamental dos estudantes universitários para o futuro sucesso do processo., ${ }^{1,5-8}$ A literatura mundial tem apontado carência educacional em transplante para estudantes de Medicina, assim como necessidade de investimento das instituições de ensino médico através de projetos de intervenção direta na comunidade acadêmica. ${ }^{6-15}$ 
No Brasil, a realidade não é diferente. A literatura científica a respeito do conhecimento dos estudantes de Medicina sobre doação e transplante de órgãos e tecidos evidencia conhecimento insuficiente e necessidade de aperfeiçoamento sobre o tema. ${ }^{8,9,11,12}$ Dentre as maiores deficiências, se encontram o conceito e diagnóstico de morte encefálica, manutenção do potencial doador, quais os órgãos e tecidos que podem ser transplantados e conhecimento insuficiente a respeito da Lei n ${ }^{\circ} 9.434$ de 04 de fevereiro de 1997 que regulamenta os transplantes de órgãos e tecidos no país.

Profissionais de saúde treinados e capazes de fazer uma adequada abordagem da família do potencial doador falecido irão influenciar positivamente na decisão de doação de órgãos. Desta forma, investir na educação dos estudantes de Medicina em transplantes é um ponto crucial que pode favorecer e influenciar no consentimento da família em doar órgãos e tecidos.? A educação de graduandos de Medicina sobre o processo de identificação de um potencial doador de órgãos e o diagnóstico de morte encefálica é um fator importante para maximizar os benefícios dos transplantes num contexto limitado de doadores de órgãos.

Sendo assim, o presente estudo se propõe a avaliar o conhecimento dos estudantes de Medicina sobre doação e transplante de órgãos, assim como identificar os possíveis fatores contrários à doação de órgãos e tecidos. Estudos como este são relevantes, pois permitem identificar áreas onde são necessárias intervenções educativas nos estudantes de Medicina, propiciando melhor qualificação daqueles futuros profissionais, além de direcionar as políticas públicas de educação em transplante.

\section{MÉTODOS}

Trata-se de um estudo de corte transversal realizado com 266 estudantes de Medicina da Escola Bahiana de Medicina e Saúde Pública (EBMSP) em Salvador, Bahia, no período de agosto a outubro de 2010. A pesquisa foi realizada com acadêmicos regularmente matriculados em turmas do primeiro ao oitavo semestres baseando-se na aplicação de questionário padronizado contendo dados demográficos e 13 questões de múltipla escolha sobre doação e transplantes de órgãos, conceito de morte encefálica, existência de aulas sobre o tema durante o curso de graduação, intenção de doar órgãos, motivos contrários a doação de órgãos, conhecimento a respeito da manutenção de um potencial doador e como deve ser feita a comunicação à família da morte de um parente. A confecção do questionário foi realizada após uma revisão da literatura científica, utilizando os bancos de dados LILACS,

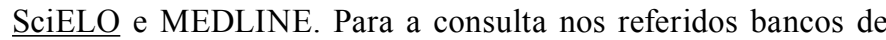
dados, utilizou-se como ponte de corte artigos publicados entre 1998 e 2010, utilizando-se os seguintes descritores: Transplantes, Morte Encefálica, Estudantes de Medicina, Doação de Órgão, Doação de Tecido.

A coleta foi feita através de visitas em salas de aulas pouco antes das atividades de ensino ou logo imediatamente após as mesmas. Cada estudante teve em média 15 minutos para responder de forma espontânea o questionário. Antes do preenchimento, os acadêmicos leram e assinaram o Termo de Consentimento Livre e Esclarecido. Esta pesquisa foi submetida à análise e aprovada pelo Comitê de Ética em Pesquisa da Escola Bahiana de Medicina e Saúde Pública com número de protocolo 039/2010.
O cálculo amostral foi realizado utilizando-se a ferramenta STATCALC do programa Epi Info versão 3.5.1, tendo como base uma população de 1000 alunos. Esse cálculo considerou que $60 \%$ dos alunos tinham conhecimento satisfatório a respeito de morte encefálica e que o resultado inesperado seria de $50 \%$. Os dados foram analisados através do programa Epi Info versão 3.5.1., procedendo-se análises de freqüência. Para verificar diferenças entre proporções e razões de prevalência, foi utilizado o teste do qui-quadrado, com intervalo de confiança de $95 \%$ (IC 95\%).

\section{RESULTADOS}

Foram entrevistados 266 estudantes de Medicina do primeiro ao oitavo semestre. A distribuição dos acadêmicos por semestre de graduação encontra-se demonstrada na Figura 1. Cinquenta e cinco por cento eram do sexo feminino, a idade mediana foi de 20,81 $\pm 1,8$ anos, variando de 17 a 34 anos. A distribuição por sexo, de acordo com o semestre se encontra descrita na Figura 2.

Figura 1 - Distribuição dos estudantes por semestre de graduação.

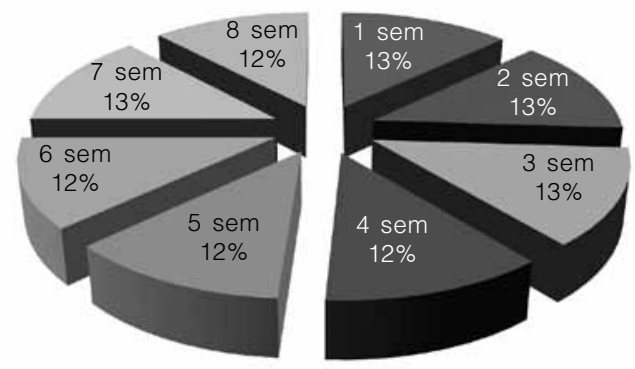

Figura 2 - Distribuição dos estudantes por sexo e semestre de graduação.

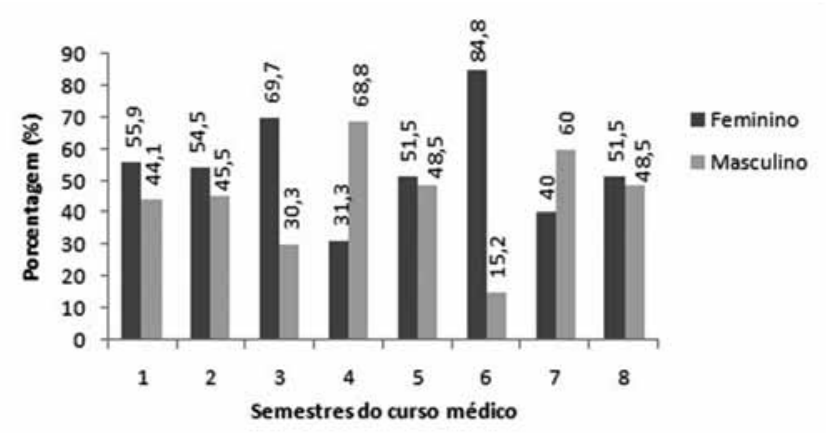

Dos graduandos que responderam ao questionário, $61,3 \%$ já tinham assistido aulas ou cursos sobre transplantes durante a graduação. Sessenta por cento dos estudantes avaliaram o seu conhecimento sobre transplantes como regular e $22,6 \%$ consideraram ruim. Os resultados da auto-avaliação podem ser observados na Figura 3.

Quando perguntados sobre quais transplantes podem ser realizados intervivos, 78,9\% responderam rim, 15,4\% não sabiam responder e 2,6\% córneas e rim. Dos transplantes que podem ser realizados sem batimentos cardíacos do doador, $53,4 \%$ dos estudantes não sabiam responder e $45,4 \%$ acertaram a questão. 
Figura 3 - Auto-avaliação do conhecimento dos estudantes de Medicina sobre doação e transplantes de órgãos.

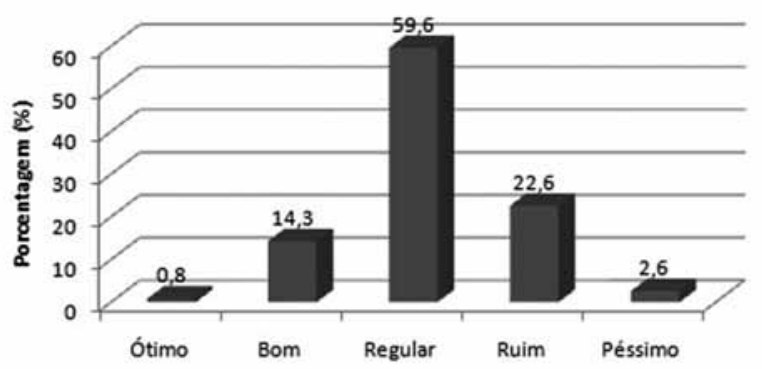

Noventa e um por cento dos alunos afirmaram saber qual o conceito de morte encefálica (ME) e 72\% tinham noções de como realizar o diagnóstico. Quando perguntados sobre o conceito de morte encefálica, $88 \%$ responderam que o indivíduo está sem função encefálica, 7,9\% responderam que morte encefálica é quando o indivíduo está em Glasgow 3. A morte encefálica foi definida por $2,3 \%$ dos estudantes como estado de coma e 1,9\% dos estudantes não sabiam responder. Analisando o cruzamento das porcentagens dos estudantes que afirmavam saber o conceito de morte encefálica e dos que realmente acertaram tal conceito através do teste do qui-quadrado, os resultados foram estatisticamente significantes $(\mathrm{p}=0,04)$. Ou seja, a maioria dos alunos que afirmou saber o conceito de morte encefálica acertou o conceito referido. $\mathrm{O}$ acerto do conceito por semestre se encontra evidenciado na Figura 4. É importante ressaltar que no ano de 2009 houve atividade educativa com os estudantes do primeiro ano da graduação sobre o assunto morte encefálica e transplantes de órgãos.

Figura 4 - Acerto do conceito de morte encefálica por semestre de graduação.

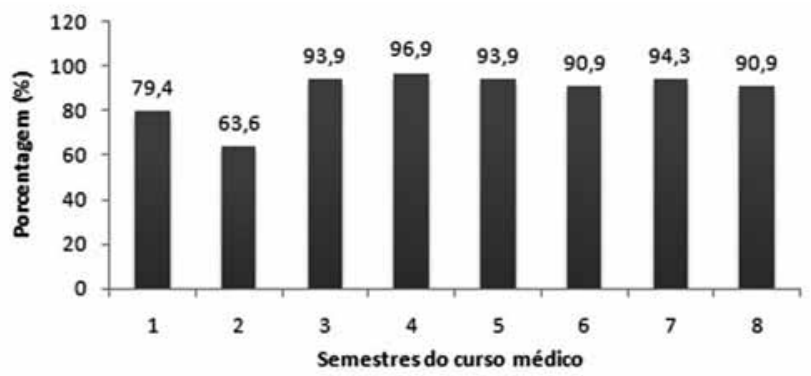

No que diz respeito à intenção de doar órgãos e tecidos, 78,6\% afirmaram que gostariam de doar, $18,8 \%$ nunca pensaram sobre o assunto e $2,6 \%$ responderam não ser doadores. Os motivos pelos quais os acadêmicos não doariam órgãos foram: $61,8 \%$ nunca pensaram sobre o assunto, $11,8 \%$ desconheciam sobre o assunto de doação e transplantes de órgãos e tecidos, 5,9\% tinham medo de doar ou simplesmente não queriam por motivos como não acreditar na justiça e honestidade do sistema de transplantes. Quatro por cento dos graduandos desconfiam de comércio de órgãos e 1,5\% nunca pensaram sobre o assunto, além de terem medo de doar órgãos e tecidos. $\mathrm{Na}$ análise de dados por semestre, observou-se que $68 \%$ dos estudantes do primeiro semestre tinham a intenção de doar órgãos e essa porcentagem foi de $94 \%$ no oitavo semestre da graduação.
No que se refere à intenção de ser doador de órgãos em transplante intervivos, 65,4\% disseram que doariam, 30,5\% não sabiam e $4,1 \%$ não seriam doadores nesse tipo de transplante. Dos que responderam "sim" ou "não sei" para doação em transplante intervivos, $42 \%$ doariam órgãos somente para parentes de primeiro grau, $43 \%$ para parentes e amigos, 9\% doariam para parentes independente do grau de parentesco e $6 \%$ doariam para parentes, amigos e desconhecidos.

No que tange aos cuidados que devem ser tomados para a manutenção do potencial doador de órgãos, 75,5\% dos estudantes desconheciam as medidas de suporte necessárias e apenas $24,5 \%$ responderam que sabiam quais cuidados deveriam ser tomados.

Por fim, quando questionados sobre como deveria ser feita a comunicação à família da morte de um parente, $50,4 \%$ responderam que sabiam como fazer a comunicação e $49,6 \%$ dos alunos não sabiam realizar tal abordagem.

\section{DISCUSSÃO}

Os resultados do presente estudo sugerem que intervenções a respeito da educação em transplantes de órgãos realizadas pela instituição de ensino pesquisada podem melhorar o conhecimento dos estudantes de Medicina sobre morte encefálica, tendo sido o acerto sobre este conceito de $88 \%$ dos estudantes pesquisados. É interessante ressaltar que os alunos do primeiro ano de graduação tiveram maior percentagem de erro em relação aos alunos do segundo ano, que tiveram atividades educativas voltadas para diagnóstico de morte encefálica e transplantes de órgãos. Essa atividade educativa provavelmente é responsável pelo alto nível de acerto dos estudantes do segundo ano, cuja porcentagem assemelha-se à dos alunos do terceiro e quarto anos. Embora a literatura científica relate a implantação e realização de projetos educacionais aplicados ao tema, ainda não há dados que quantifiquem o resultado da intervenção dos mesmos sobre o conhecimento de estudantes de Medicina. ${ }^{6,7,14}$

Ainda em relação ao conceito de morte encefálica, apesar da maioria dos acadêmicos conhecer tal conceito, $28 \%$ afirmaram não saber como fazer o diagnóstico. Ressalta-se que a amostra analisada foi constituída por alunos que ainda não ingressaram no internato, e a realização de estágios em Unidades de Terapia Intensiva (UTI) poderia ajudar os estudantes a vivenciar o processo de identificação de potencias doadores, além de dar oportunidade ao aluno a participação na condução de um diagnóstico de morte.

Em se tratando de quais transplantes podem ser realizados intervivos, tanto alunos dos primeiros semestres quantos de semestres mais avançados tiveram alto grau de acerto. No entanto, quando questionados sobre quais transplantes podem ser realizados sem batimentos cardíacos do doador, menos da metade dos entrevistados acertaram a questão, ressaltando a necessidade de intervenção na área referida.

O trabalho também identificou a necessidade de intervenção educativa em relação aos cuidados necessários para manter um potencial doador. Menos de $25 \%$ dos estudantes sabiam como realizar as medidas de suporte na manutenção de um potencial doador; no entanto, cabe salientar que a amostra pesquisada não incluiu alunos do internato que apresentam maior contato com assuntos relacionados à Medicina Intensiva. Maia et al. ${ }^{11}$ ressaltam que os cuidados intensivos para manutenção do potencial doador 
são imprescindíveis para a boa qualidade dos órgãos e tecidos e para a realização de transplantes. No mesmo trabalho, afirmam que alguns estudantes da área de saúde apresentam conhecimento insuficiente e superficial sobre o tema. Deve- se, então, investir em matérias que proporcionem aos estudantes maior conhecimento em fisiologia e fisiopatologia da morte encefálica e interpretação de exames laboratoriais. Tais conhecimentos devem ser disseminados entre os estudantes, pois é através do desenvolvimento de habilidades nas áreas referidas, que os acadêmicos se sentirão mais preparados para monitorização e realização de cuidados intensivos destinados ao potencial doador. ${ }^{11}$

O presente estudo aponta que a maioria dos acadêmicos, $78,6 \%$, é a favor da doação de órgãos. Esse resultado é semelhante a alguns estudos da literatura que demonstram que a maioria dos estudantes de Medicina tem intenção de doar órgãos. ${ }^{9,10,16,17}$ A literatura científica também sugere que a decisão de doar órgãos por parte dos estudantes, futuros profissionais de saúde, influencia positivamente as famílias dos potencias doadores de órgãos. ${ }^{1,7,8}$

Menos de 22\% dos estudantes de Medicina nunca pensaram em doar órgãos ou não tinham intenção de doar. Os maiores motivos para essas escolhas foram: nunca pensaram sobre o assunto ou desconhecimento sobre o mesmo. Todavia, observa-se que essa porcentagem estava associada aos primeiros semestres, quando havia pouco conhecimento dos acadêmicos sobre o assunto de doação de órgãos para transplantes.

Sessenta e oito por cento dos estudantes do primeiro semestre têm intenção em doar órgãos, e essa porcentagem aumenta para $94 \%$ nos alunos do oitavo semestre da graduação. No entanto, os resultados do trabalho de Galvão et al. ${ }^{9}$ evidenciam que quanto mais velhos os estudantes de Medicina, menor a intenção de doar órgãos. Pode ser que intervenções didáticas sobre transplante de órgãos e tecidos realizadas no ano de 2009, na EBMSP, somado ao maior conhecimento a respeito do tema no decorrer da graduação estejam influenciando positivamente o aumento da intenção de doar da população estudada. Isso sugere que o conhecimento adquirido na graduação de Medicina pode influenciar na decisão do acadêmico sobre doação de órgãos, levantando a hipótese de que, através da educação da população em doação de órgãos para transplantes e conceito de morte encefálica, a intenção de doar órgãos e tecidos possa aumentar proporcionalmente ao grau de educação nas áreas referidas, como sugerem os trabalhos de intervenção nos cursos das escolas médicas. ${ }^{1,7,8}$
A respeito da auto-avaliação dos acadêmicos sobre o conhecimento em doação e transplantes de órgãos, $60 \%$ dos estudantes avaliaram seu conhecimento como regular, $22,6 \%$ consideraram ruim e menos de $1 \%$ definiu como ótimo. Esses dados corroboram a literatura ${ }^{9} \mathrm{e}$ evidenciam a importância da incorporação de temas relacionados à doação e transplante de órgãos no currículo das escolas médicas, a fim de educar futuros profissionais de saúde para que estes diminuam a discrepância entre o pequeno número de potencias doadores e a quantidade de receptores no país. . $^{1,8}$

Existem grandes oportunidades para diminuir a fila de espera para transplantes no Brasil. ${ }^{4}$ Investir nos futuros profissionais de saúde é um dos caminhos para atingir essa meta. Dessa forma, projetos educacionais devem ser criados para que os estudantes possam melhorar o nível de conhecimento e influenciar positivamente a taxa de transplantes no país. A implantação de cursos e matérias sobre doação de órgãos e tecidos para transplante no currículo das escolas médicas e o incentivo a estágios nessa área são alternativas já testadas e que vêm dando certo em faculdades brasileiras e internacionais. ${ }^{6,7,11,14,15}$ Educar precocemente os estudantes de Medicina é uma alternativa direta que contribui para aumentar o número de transplantes em escala mundial.

\section{CONCLUSÃO}

Foi possível concluir que a maioria dos estudantes de Medicina da amostra estudada apresentou conhecimento (88\%) sobre morte encefálica, apesar de $60 \%$ dos alunos avaliarem seus conhecimentos como regular.

Os dados deste trabalho também evidenciam que no decorrer do curso médico, os estudantes vão adquirindo maior conhecimento sobre transplante e doação de órgãos e tecidos, aumentando a intenção de doar. Dessa forma, o presente trabalho sugere que a educação sobre o tema pode melhorar as taxas de doação de órgãos. É fundamental educar estudantes de Medicina, para que os futuros profissionais sejam capacitados a identificar e manter um potencial doador, conduzir o diagnóstico de morte encefálica e melhorar as taxas de consentimento familiar para doação de órgãos e tecidos, contribuindo para o aumento do número de transplantes. Dessa forma, é necessária a inserção de matérias ou cursos específicos de doação de órgãos e tecidos para transplantes no currículo das faculdades de Medicina.

\section{ABSTRACT}

Purpose: To assess knowledge of medical students on organ donation and transplantation, the concept of brain death, and their ability in identifying contrary factors to the organ and tissue donation. Methods: This is a cross-sectional study conducted with 266 medical students from the first to fourth grades of the Medical school. Data collection was conducted between August and October 2010. We have applied a questionnaire containing demographic data and 13 multiple choice questions on the subject. Results: Among respondents, $61.3 \%$ had educational activities on organ transplantation during the Medical course. Sixty percent of students ranked their knowledge on the topic as regular. Eighty-eight percent of students understood the concept of brain death, and $72 \%$ had notions about the diagnosis. When asked about what organs can be transplanted in case of living donors, $78.9 \%$ answered correctly. As to transplants performed under no heartbeating, 45.4\% answered the question, and 53.4\% was not aware of them. When asked on the intention to donate organs and tissues, $78.6 \%$ were donors, and the analysis per semester showed a progressive increase in the willingness to donate. As to the intensive care for the maintenance of potential organ donors, $75.5 \%$ of students were unaware on the supportive measures required. Regarding to the 
communication to the family about the death of a relative, $50.4 \%$ knew how to make such approach. Conclusion: We concluded that the majority of medical students in the sampling studied had knowledge on brain death. During the medical course, students increase their knowledge about transplantation and donation of organs and tissues. This knowledge increases the intention to donate, suggesting that education is essential to improve rates of organ donation. Thus, it is necessary to insert specific matters or courses on the organ and tissue transplants in the medical school curriculum

Keywords: BK Virus; Kidney Transplantation; Immunosuppression

\section{REFERÊNCIAS}

1. Schaeffner ES, Windisch W, Freidel K, Breitenfeldt K, Winkelmayer WC. Knowledge and attitude regarding organ donation among medical students and physicians. Transplantation. 2004 Jun;77(11):1714-8.

2. Cantarovich F. Improvement in organ shortage through education. Transplantation 2002 Jun;73(11):1844-6.

3. Ministério da Saúde [homepage na Internet]. Brasil: Lista de Espera (Ativos e semiativos) - Total 2009 [Atualizado em 2009; acesso em 13 de Out de 2010]. Disponível em: http://portal.saude.gov.br/portal/arquivos/pdf/Lista_de_Espera_2009.pdf

4. Marinho A. Um estudo sobre as filas para transplantes no Sistema Único de Saúde brasileiro. Cad. Saúde Pública. 2006 Out;22(10):2229-39.

5. Essman C, Thornton J. Assessing medical student knowledge, attitudes, and behaviors regarding organ donation. Transplant Proc. 2006 Nov;38(9):2745-50.

6. Manyalich M, Paredes D, Ballesté C, Menjívar A. The PIERDUB project: International Project on Education and Research in Donation at University of Barcelona: training university students about donation and transplantation. Transplant Proc. 2010 Jan-Feb;42(1):117-20.

7. Garcia CD, Barboza AP, Goldani JC, Neumann J, Chem R, Camargo J, et al. Educational program of organ donation and transplantation at medical school. Transplant Proc. 2008 May;40(4):1068-9.

8. Oliveira Junior RE, Saldanha BO, Oliveira APP, Santos EA, Oliveira MP, Pereira WA, et al. Conhecimento sobre morte encefálica e doação de órgão entre estudantes de Medicina de Belo Horizonte. JBT J Bras Transpl. 2009; Jul-Set:12(3):1149-53.

9. Galvão FHL, Caires RA, Azevedo-Neto R, Mory EK, Figueira ERR, Otsuzi TS, et al. Conhecimento e opinião de estudantes de Medicina sobre doação e transplante de órgãos. Rev Assoc Med Bras 2007 Oct;53(5):401-6.

10. Dutra MMD, Bonfim TAS, Pereira IS, Figueiredo IC, Dutra AMD, Lopes AA. Knowledge about transplantation and attitudes toward organ donation: a survey among medical students in Northeast Brazil. Transplant Proc. 2004 May;36(4):818-20.

11. Maia BO, Amorim JS. Morte encefálica: conhecimento de acadêmicos de enfermagem e Medicina. JBT J Brás Transpl. 2009 Abr-Jun;12(2): 1088-91.

12. Espínola RF, Rodrigues BA, Penteado LT, Tan-Ho Gisela, Gozzan JOA, Freitas JAH. Conhecimento dos estudantes de Medicina sobre doação de córneas. Arq Bras Oftalmol. 2007;70(4):581-4.

13. Bardell T, Hunter DJ, Kent WD, Jain MK. Do medical students have the knowledge needed to maximize organ donation rates? Can J Surg. 2003 Dec;46(6):453-7.

14. Garcia CD, Goldani JC, Neumann J, Chem R, Chem E, Camargo JJ, et al. Importância do programa educacional de doação e transplante em escolas médicas. JBT J Bras Transpl. 2009 Jan-Mar;12(2):1049-51.

15. Essman CC, Lebovitz DJ. Donation education for medical students: enhancing the link between physicians and procurement professionals. Prog Transplant. 2005 Jun;15(2):124-8.

16. Chen JX, Zhang TM, Lim FL, Wu HC, Lei TF, Yeong PK, et al. Current knowledge and attitudes about organ donation and transplantation among Chinese university students. Transplant Proc. 2006 Nov;38(9):2761-5.

17. Strenge H. Organ donation from the viewpoint of the medical students. Psychother Psychosom Med Psychol. 1998 Nov;48(11):457-62. 